\title{
Coating-free mirrors for high precision interferometric experiments
}

\author{
Stefan Goßler,* Jeff Cumpston, Kirk McKenzie, Conor M. Mow-Lowry, Malcolm B. Gray, and David E. McClelland \\ Centre for Gravitational Physics, Department of Physics, Faculty of Science, The Australian National University, Canberra, \\ Australian Capital Territory 0200, Australia
}

(Received 27 July 2007; published 8 November 2007)

\begin{abstract}
Thermal noise in mirror optical coatings may not only limit the sensitivity of future gravitational-wave detectors in their most sensitive frequency band but is also a major impediment for experiments that aim to reach the standard quantum limit or cool mechanical systems to their quantum ground state. We present the design and experimental characterization of a highly reflecting mirror without any optical coating. This coating-free mirror is based on total internal reflection and Brewster-angle coupling. In order to characterize its performance, the coating-free mirror was incorporated into a triangular ring cavity together with a high quality conventional mirror. The finesse of this cavity was measured using an amplitude transfer function to be about $\mathcal{F} \simeq 4000$. This finesse corresponds to a reflectivity of the coating-free mirror of about $R \simeq 99.89 \%$. In addition, the dependence of the reflectivity on rotation was mapped out.
\end{abstract}

DOI: $10.1103 /$ PhysRevA.76.053810

PACS number(s): 42.79.Fm, 42.60.Da, 42.50.-p, 05.40.Jc

\section{INTRODUCTION}

The optical quality of mirror coatings has vastly improved over the last decade, driven by high precision optical metrology applications. One such application is the detection of gravitational waves (GWs) using interferometry. The performance of planned GW detectors in their most sensitive frequency band is, however, predicted to be limited not by optical properties but by mechanical properties responsible for thermal noise in the coatings. For a detailed analysis of thermal noise related to optical coatings, see, e.g., [1-6].

Coating thermal noise is also a major impediment to experiments aimed at reaching and eventually even surpassing the so-called standard quantum limit (SQL) [7]. The SQL is a direct consequence of the Heisenberg uncertainty principle. The SQL of an interferometric measurement arises from quantum fluctuations in the amplitude and phase quadratures of the interrogating laser field, commonly referred to as radiation-pressure noise and shot noise, respectively.

Due to the different scalings of shot noise and radiationpressure noise with power, at a given frequency there exists a power level at which the two are equal in magnitude. The sensitivity reached under these conditions is the SQL. Despite the effort of numerous groups, the SQL has never been reached for the measurement of a macroscopic object such as the mirror of an interferometer [8-10].

Closely related to SQL experiments are attempts to cool macroscopic objects to their quantum ground state $[11,12]$, as well as experiments aiming to generate quantum mechanical entanglement between macroscopic objects [13]. Careful analyses show that again coating thermal noise must be greatly reduced in order to observe quantum mechanical phenomena with objects visible to the naked eye. In [14] it was shown that coating thermal noise can also set a limit to the frequency stability of a laser when using a rigid cavity as the reference.

\footnotetext{
*Present address: Max-Planck-Institut für Gravitationsphysik, Albert-Einstein-Institut, Callinstrasse 38, D-30167 Hannover, Germany.
}

A possible way to overcome this limitation is to improve the mechanical properties of the optical coatings. This is currently being tried by adding various dopants, e.g., titania, to the coating materials. Another promising approach is to reduce the thickness of the coatings by, for example, using grating waveguide structures. Waveguide coatings are already used as wavelength-selective elements and as broadband mirrors at $1550 \mathrm{~nm}$ laser wavelength. The demonstration of a highly reflecting grating waveguide coating at 1064 $\mathrm{nm}$ is, however, still to be done [15].

Khalili [16] suggested reducing coating thermal noise in a GW detector by replacing the end mirrors with short FabryPérot cavities. By using a front mirror of moderate reflectivity in this Fabry-Pérot cavity, a thinner coating can be used, reducing the coating thermal noise by a substantial factor. This approach is, however, impractical for use in other applications.

A more radical approach is to construct highly reflecting mirrors with no optical coatings. In 2004 Braginsky and Vyatchanin suggested using corner reflectors as end mirrors in advanced gravitational-wave detectors [17]. These corner cubes rely on total internal reflection (TIR) but need an antireflection coating on the curved input face. As an antireflection coating can be much thinner than a highly reflecting coating, its thermal noise should be much smaller than that of a highly reflecting coating. This system is designed for use with large-diameter laser beams $(\approx 10 \mathrm{~cm})$ so that optical loss accompanying reflection off the corner discontinuity will be very small. However, small-diameter laser beams as used in SQL and optical cooling type experiments would read out the discontinuity and suffer unacceptably large optical loss.

In 2006, Cella and Giazotto [18] suggested another corner-reflector-based design. His method relies on the interference of the beam promptly reflected at the front face with the beam being reflected off the rear face of the corner reflector. This is the most complex of the suggested designs, as it relies on the front face curvature of the reflector very precisely matching the curvature of the beam's phase fronts as well as on a very high homogeneity of the refractive index of 

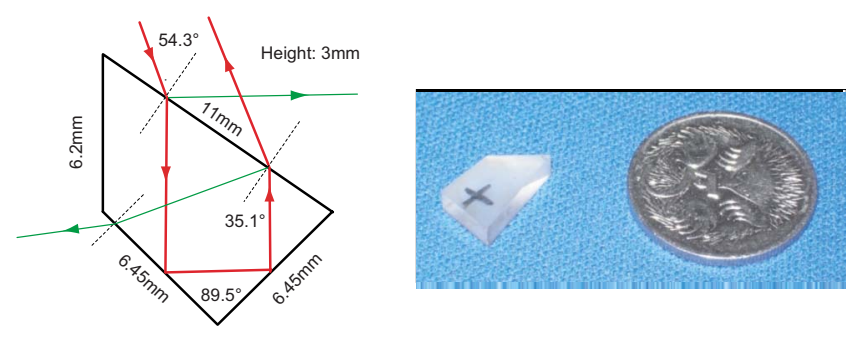

FIG. 1. (Color online) Left: Sketch of the CFM with all dimensions. Right: Photograph of a coating-free mirror alongside an Australian five cent coin.

the substrate material to ensure constructive phase wrapping at another mirror to form a cavity.

In this paper, we introduce a coating-free mirror (CFM) also based on a corner reflector using total internal reflection, but without reflection incorporating the discontinuity at the corner. Furthermore, the need for antireflection coating is avoided by coupling the laser beam into the corner cube at the Brewster angle. In the next section, we present a detailed analysis of this design. This is followed by a description and results from an experimental prototype and future outlook.

\section{COATING-FREE MIRROR DESIGN}

The CFM is shown in Fig. 1. We will describe the principle of operation in the context of our objective, which was to design a CFM that had a high reflectivity (of the order of 99.9\%) and was light enough to be used in SQL and optical cooling experiments. Such experiments aim to use gram scale mirrors. Hence, the CFM was designed to be as small as possible without being limited by diffraction loss. The CFM dimensions were chosen such that each relevant face was at least three times the diameter of the incident laser beam plus some margin to allow for alignment. This consideration and the geometry of the test cavity led to the CFM dimensions shown in Fig. 1. The laser beam enters the CFM via the Brewster angle and is then reflected twice at the rear faces using TIR. In this way, the discontinuity of the corner is avoided. The beam is then coupled out of the CFM via the Brewster angle. The sketch shows this main beam path along with the two residual reflections at the front face due to imperfect Brewster-angle coupling. With the given dimensions and a density of Suprasil 1 of $2.20 \mathrm{~g} / \mathrm{cm}^{3}$, the weight of the CFM was $0.43 \mathrm{~g}$. tion

The angle $\Theta_{c}$ above which TIR occurs satisfies the rela-

$$
\Theta_{c}=\sin ^{-1} \frac{n_{1}}{n_{2}} \text { for } n_{1}>n_{2}
$$

with $n_{i}$ giving the refractive indices of two media with a common interface. As can be seen from Eq. (1) it is evident that TIR can occur only for a reflection at an optically less dense medium. In other words, the laser beam has to hit the TIR faces from inside the CFM substrate. This requires the beam to enter and consequently to leave the substrate, placing two requirements on the CFM: First the internal trans-

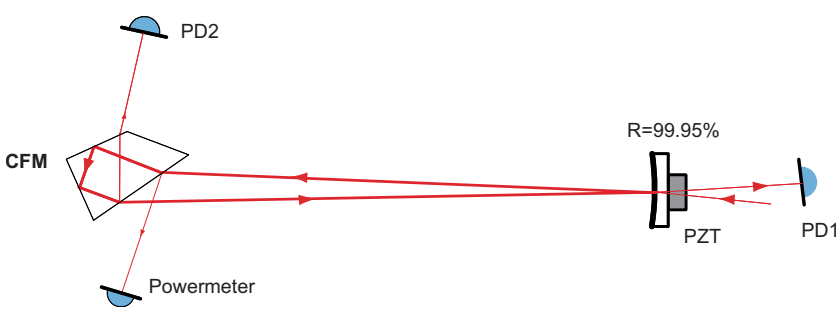

FIG. 2. (Color online) Setup to characterize the CFM reflectivity (not to scale).

mission loss due to absorption and scatter must be small enough and second the in- and outcoupling of the beam must be done with small enough residual reflection.

Coupling of the beam into and out of the CFM at the Brewster angle allows, in principle, lossless coupling of the laser. However, due to the cavity geometry (see Fig. 2) it was required that the incident and outgoing beams meet at about $7 \mathrm{~cm}$ from the CFM. To accommodate this requirement the corner between the TIR surfaces was made slightly less than $90^{\circ}$. This deviation from a right angle leads to a deviation from perfect Brewster angle at the front face for both the incident and outgoing beams. As a consequence, a fraction of the light power is reflected at these two points, as illustrated in Fig. 1. By separately adjusting the angles of the input and output beams on the front face of the CFM (that is, putting in a "kink") one could correct for the deviation and thus minimize the residual reflection. However, the loss due to imperfect Brewster-angle coupling is small in our case. Using Snell's law, the refractive index of Suprasil 1 of $n=1.45$, and the angle of incidence of $\theta_{\text {in }}=54.3^{\circ}$ for the incident and $\theta_{\text {out }}=35.1^{\circ}$ for the outgoing beam, one obtains a reflection loss of $\alpha_{\text {refl }}=0.021 \%$. Also these two beams can be used for diagnostics.

A further source of loss is the transmission loss of the CFM. With the transmissivity as specified by the manufacturer and $1.5 \mathrm{~cm}$ optical pathlength inside the CFM, the internal transmission loss of the CFM was calculated to be $\alpha_{\text {trans }}=0.006 \%$. Under the assumption that TIR is perfect, the overall optical loss of the CFM is thus $\alpha_{\mathrm{CFM}}=0.027 \%$, allowing a theoretical reflectivity of $R \simeq 99.97 \%$.

\section{EXPERIMENTAL SETUP}

Characterization of the CFM was performed by incorporating it into a cavity. The other cavity mirror was a spherical (radius of curvature $\mathrm{R}=0.5 \mathrm{~m}$ ) high quality conventional mirror with a reflectivity of $R=99.95 \%$. The conventional mirror was mounted on a piezoelectric transducer (PZT) to enable microscopic length control of the cavity by a servo system. Figure 2 shows the setup used. The pitch adjustment of the CFM was done via a micrometer such that the reflected beam was aligned antiparallel with respect to the incident beam. From a geometrical analysis, it is evident that a lateral shift of the incident beam shifts the longitudinal position at which it crosses the reflected beam. Thus, the initial cavity length could be manually fine-adjusted by means of a transverse shift of the steering mirrors before the cavity. 
Once resonant peaks could be observed when scanning the length of the cavity via the PZT, the incident beam could be accurately matched to the cavity eigenmode regarding both the angular alignment and the cavity length. The round trip length of the cavity was $142 \mathrm{~mm}$. The incident light power was $4 \mathrm{~mW}$. The cavity was stabilized to the laser frequency using the Pound-Drever-Hall technique [19]. The control signals were derived from the light reflected by the resonator and incident on photodiode PD1. The light from the two residual reflections was used for diagnostics, e.g., to monitor the intracavity power.

In order to obtain accurate information about the relevant cavity properties, amplitude modulation (AM) transfer functions were recorded. By modulating amplitude sidebands at different Fourier frequencies onto the beam incident on the cavity, the frequency and phase response of the cavity can be mapped out. In practice, a swept sine function was used to sweep through all relevant Fourier frequencies. The transmitted, or intracavity, light contains a fraction of the sideband power at a given Fourier frequency according to the cavity response at this particular frequency. By demodulating the signal from photodiode PD2 in Fig. 2 with the modulation frequency, the intracavity sideband power at all Fourier frequencies was determined and normalized to the incident sideband power. The half width at half maximum (HWHM) of the cavity was determined from the Fourier frequency at which the intracavity sideband power was down by $3 \mathrm{~dB}$, or equivalently the sideband phase lagged by $45^{\circ}$.

From the linewidth of the cavity, its length, and the known reflectivity of the conventional mirror, the reflectivity of the CFM was inferred.

\section{RESULTS}

Figure 3 shows the data obtained (solid line) using AMsideband cavity interrogation for the CFM rotational alignment angle (alignment with respect to a vertical axis through the CFM) that corresponds to maximum observed CFM reflectivity. The rotational angle was set manually via a calibrated adjustment knob at the CFM mount. In order to obtain the alignment angle of maximum CFM reflectivity, the rotational alignment was iteratively altered until the cavity linewidth went through its minimum. The reflectivity of the CFM was then used as the only fitting parameter in a leastsquares fit that is also shown in Fig. 3 (dashed line). The 3 $\mathrm{dB}$ point as well as the corresponding $45^{\circ}$ point are highlighted in the graphs. The full cavity linewidth (full width at half maximum) for this rotational alignment angle of the CFM is $560 \mathrm{kHz}$. The corresponding cavity finesse is $\mathcal{F}=3930 \pm 260$. The reflectivity of the CFM as obtained from the least-squares fit is $R=99.89 \% \pm 0.01 \%$.

Since the theoretical value for the CFM reflectivity differed from the experimental value, it is evident that an optical loss mechanism that was previously not taken into account dominates the loss. When the room lights were switched off and an infrared viewer was used, it was clear that the two TIR surfaces were strong sources of scattered light. The surfaces of the front and the two TIR faces of the CFM were polished to $\lambda / 10$ quality. From the experiment it

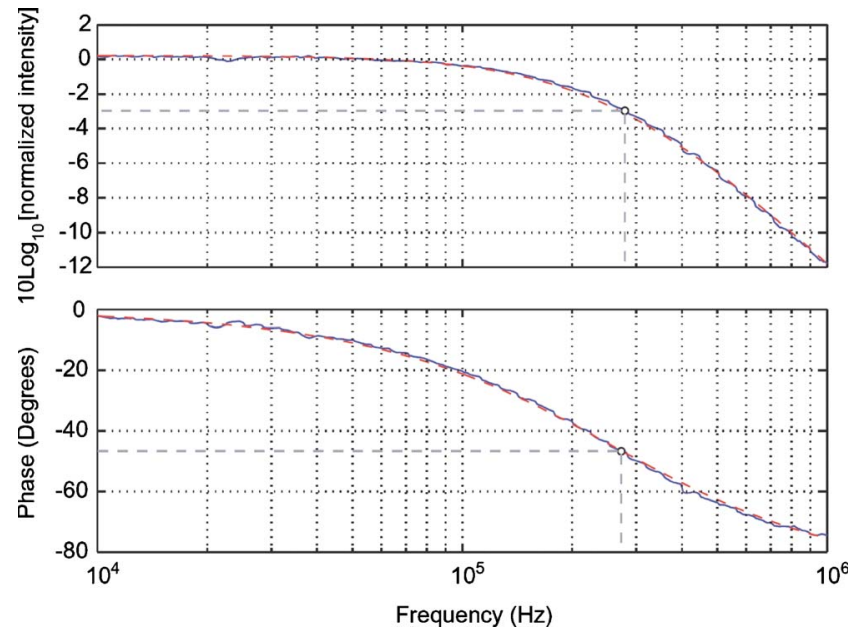

FIG. 3. (Color online) Measured frequency (top graph) and phase (bottom graph) response of the cavity for the optimum rotational alignment angle of the CFM. The solid line represents the measured data while the dashed line is the result of a least-squares fit used to determine the reflectivity of the CFM. The $-3 \mathrm{~dB}$ point, corresponding to half the cavity linewidth (HWHM), of the frequency response is marked along with the equivalent $45^{\circ}$ phase point.

is evident that this surface quality is not compatible with a reflectivity in excess of $99.9 \%$. A numerical investigation of TIR scattering due to random surface roughness was undertaken in [20], though none of the cases investigated therein provide numerical values for a surface roughness corresponding to that of the polished CFM surfaces. Surface roughness was also attributed to be the main source of loss in a fused silica monolithic TIR resonator investigated in [21]. According to [22,23], it is possible to reduce the effects of this phenomenon dramatically by superpolishing the reflective surfaces to rms values in the subangstrom regime, where TIR reflects $99.9999 \%$ of the power.

As mentioned above, the coupling of the beam into and out of the CFM was not done exactly at Brewster's angle. When the rotational alignment of the CFM was changed by a small amount, then one beam was closer to the Brewster angle while the other beam moved farther away from the Brewster angle. This feature led to a rather flat characteristic of the CFM reflectivity when rotated away from optimum alignment. The measurements presented in Fig. 4 were obtained via AM transfer function measurements for different rotational alignment angles of the CFM. The solid line is normalized to meet the obtained maximum reflectivity of the CFM. This normalization had to be done since the experimentally obtained value of the CFM reflectivity was, as mentioned above, found to be lower than the theoretical value.

It should be noted that the CFM is in principle a retroreflector and hence the cavity alignment does not, to first order, suffer from a small rotational misalignment of the CFM. An accurate geometrical analysis revealed, however, that the point at which the incident and outgoing beams converge shifts along the cavity axis as the CFM rotates. Hence, the point at which the input and output beams converge is no longer at the surface of the conventional mirror. This results 


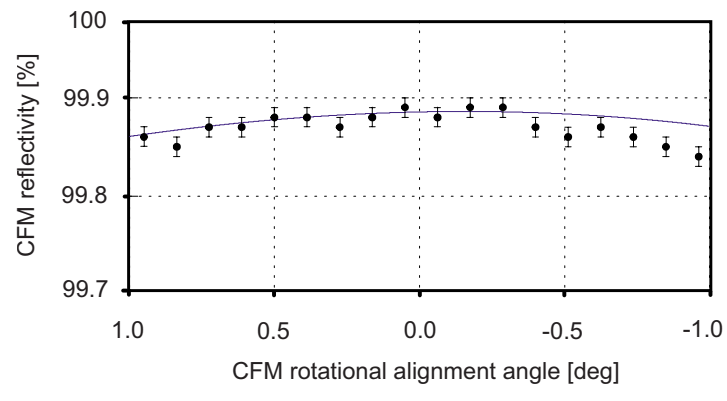

FIG. 4. (Color online) Reflectivity of the CFM versus its rotational alignment angle. The error bars arise from the relative calibration of the power meter used. Error bars in the rotational angle are too small to be displayed. The solid line represents the calculated change of reflectivity of the CFM due to deviation from the Brewster angle of the in- and outcoupled beams. The calculated curve is normalized in such a way that its maximum coincides with the maximum reflectivity of the CFM as experimentally obtained.

in the respective beam positions being slightly offset at this point, and part of the circulating power does not reproduce as desired. As this effect leads to a slightly reduced modematching quality but not to a change of cavity finesse, no corrective action was taken. However, it was evident that it caused a reduction in circulating power with CFM rotation in addition to that due to the change in CFM reflectivity. This was investigated by probing the resonant intracavity power with measurements of the beam reflected from the front face of the CFM (the beam hitting the power meter in Fig. 2). This beam gives the cavity power for a given CFM rotation angle, weighted by the rotationally dependent reflectivity of the front face. This is compared in Fig. 5 to a calculation of the power this beam would have if the change in reflectivity of the Brewster window was solely responsible for changes in intracavity power. As such, the difference between this calculated curve and the measured power represents power lost due to misalignment.

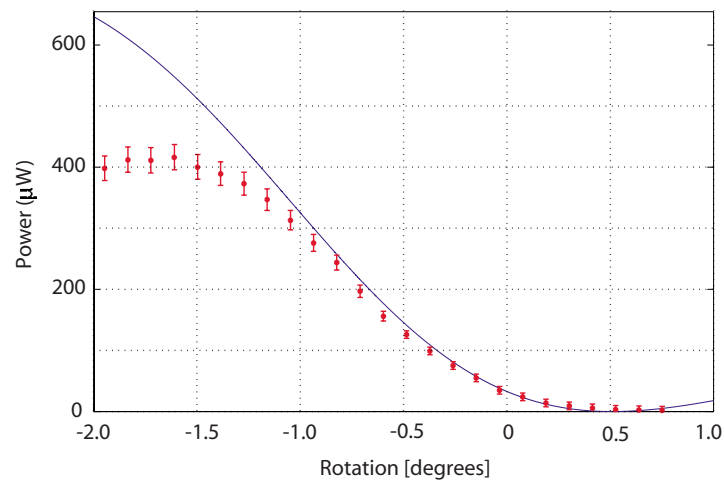

FIG. 5. (Color online) Power reflected from the front face of the CFM (the beam hitting the power meter in Fig. 2) as a function of rotational alignment. The solid line is derived under the assumption that no cavity mismatch occurs, and changes in intracavity power are caused only by deviations of the beams from the Brewster angle at the CFM front face. Thus, the difference between this curve and the experimental values corresponds to the power lost from the $\mathrm{TEM}_{00}$ mode.

\section{FUTURE WORK}

In follow-up experiments we will increase the CFM reflectivity. According to [22] the loss due to scattering at the TIR surfaces can be reduced by reducing the surface roughness via superpolishing. This can provide a reflectivity of $99.9999 \%$ from these surfaces [23]. The loss of the beams inand outcoupled to the CFM can be reduced by altering the front face of the CFM such that both beams are incident without deviating from the Brewster angle. By combining these two improvements, the CFM promises to be limited by internal transmission loss only.

Although no photothermal effects were observed in the CFM this can become problematic when operating with higher input powers. If the need arises, a CFM can be made from Suprasil SV311 grade fused silica, which can have an absorption as low as $0.2 \mathrm{ppm} / \mathrm{cm}$, more than one order of magnitude less than Suprasil 1 as used for the CFM described here. The same consideration applies for thermal lensing inside the CFM.

In order to fully benefit from the use of CFMs in SQL and cooling type experiments, it would be desirable to create an all-coating-free cavity. Such a cavity consisting of two CFMs as described in this paper would be an unstable plane-plane cavity. A stable cavity could be made, for example, by polishing a curvature into one of the TIR faces of one of the CFMs. However, as this would introduce astigmatism, a corrective cylindrical curvature would have to be overlaid on the curved surface.

A coating-free beam splitter can be realized using evanescent coupling, or frustrated total internal reflection [24]. This combined with CFMs would then enable the construction of a range of coating-free interferometers.

\section{CONCLUSION}

We have presented a unique design for a CFM for use in optomechanical experiments aimed at reaching quantum limits. The mirror is based on TIR at the rear sides of a low loss subtrate. In contrast to previous suggestions, the readout of the discontinuity at the corner of the CFM is avoided by using two reflections. The laser beam is coupled into and out of the CFM via Brewster-angle transmission, thus avoiding the need for antireflection coatings.

To characterize the first prototype mirror of this design, it was incorporated into a triangular ring cavity. A finesse $\mathcal{F}$ $\simeq 4000$ was achieved. Due to the resulting deviation of the in- and outcoupled laser beams from the Brewster angle, the reflectivity of the CFM depends on rotation. This dependence was mapped out and found to be in good agreement with theory for misalignment angles smaller than $\pm 1^{\circ}$. The maximum reflectivity of the CFM was found to be about $R$ $\simeq 99.89 \%$. At an input light power of $4 \mathrm{~mW}$, no photothermal effects were observed.

\section{ACKNOWLEDGMENT}

This work was supported by the Australian Research Council under the auspices of the Australian Consortium for Interferometric Gravitational Astronomy. 
[1] G. M. Harry, A. M. Gretarsson, P. R. Saulson, S. E. Kittelberger, S. D. Penn, W. J. Startin, S. Rowan, M. M. Fejer, D. R. M. Crooks, G. Cagnoli, J. Hough, and N. Nakagawa, Class. Quantum Grav. 19, 897 (2002).

[2] G. M. Harry, H. Armandula, E. Black, D. R. M. Crooks, G. Cagnoli, J. Hough, P. Murray, S. Reid, S. Rowan, P. Sneddon, M. M. Fejer, R. Route, and S. Penn, Appl. Opt. 45, 1569 (2006).

[3] S. D. Penn, P. H. Sneddon, H. Armandula, J. C. Betzwieser, G. Cagnoli, J. Camp, D. R. M. Crooks, M. M. Fejer, A. M. Gretarson, G. M. Harry, J. Hough, S. C. Kittelberger, M. J. Mortonson, R. Route, S. Rowan, and C. C. Vassiliou, Class. Quantum Grav. 20, 2917 (2003).

[4] M. M. Fejer, S. Rowan, G. Cagnoli, D. R. M. Crooks, A. Gretarsson, G. M. Harry, J. Hough, S. D. Penn, P. H. Sneddon, and S. P. Vyatchanin, Phys. Rev. D 70, 082003 (2004).

[5] D. R. M. Crooks, G. Cagnoli, M. M. Feijer, A. Gretarsson, G. Harry, N. Nakagawa, S. Penn, R. Route, S. Rowan, and P. H. Sneddon, Class. Quantum Grav. 21, S1059 (2004).

[6] S. Rowan and J. Hough, Phys. Lett. A 347, 25 (2005).

[7] V. B. Braginsky and F. Y. Khalili, Quantum Measurement (Cambridge University Press, Cambridge, U.K., 1995).

[8] C. M. Mow-Lowry, S. Goßler, B. J. J. Slagmolen, J. Cumpston, M. B. Gray, and D. E. McClelland, J. Phys.: Conf. Ser. 32, 362 (2006)

[9] I. Tittonen, G. Breitenbach, T. Kalkbrenner, T. Müller, R. Conradt, N. Blanc, N. F. de Rooij, E. Steinsland, and S. Schiller, Phys. Rev. A 59, 1038 (1999).

[10] V. B. Braginsky, F. Y. Khalili, and P. S. Volikov, Phys. Lett. A
287, 31 (2001).

[11] T. Corbitt, Y. Chen, H. Mueller-Ebhardt, E. Innerhofer, D. Ottaway, H. Rehbein, D. Sigg, S. Whitcomb, C. Wipf, and N. Mavalvala, Phys. Rev. Lett. 98, 150802 (2007).

[12] O. Arcizet, P.-F. Cohadon, T. Briant, M. Pinard, and A. Heidmann, Nature (London) 444, 71 (2006).

[13] H. Mueller-Ebhardt, H. Rehbein, R. Schnabel, K. Danzmann, and Y. Chen, arXiv:quant-ph/0702258.

[14] K. Numata, A. Kemery, and J. Camp, Phys. Rev. Lett. 93, 250602 (2004).

[15] A. Bunkowski, O. Burmeister, D. Friedrich, K. Danzmann, and R. Schnabel, Class. Quantum Grav. 23, 7297 (2006).

[16] F. Ya. Khalili, Phys. Lett. A 334, 67 (2005).

[17] V. B. Braginsky and S. Vyatchanin, Phys. Lett. A 324, 345 (2004).

[18] G. Cella and A. Giazotto, Phys. Rev. D 74, 042001 (2006).

[19] R. W. P. Drever, J. L. Hall, F. V. Kowalski, J. Hough, G. M. Ford, A. J. Munley, and H. Ward, Appl. Phys. B: Photophys. Laser Chem. 31, 97 (1983).

[20] M. Nieto-Vesperinas and J. A. Sanchez-Gil, J. Opt. Soc. Am. A 9, 424 (1992).

[21] S. Schiller, I. I. Yu, M. M. Fejer, and R. L. Byer, Opt. Lett. 17, 378 (1992).

[22] N. J. Brown, Annu. Rev. Mater. Sci. 16, 371 (1986).

[23] J. W. Hudgens, Environmental Sciences Management Program Project No. 60231 (NIST, Gaithersburg, MD, 1998).

[24] F. Albiol, S. Navas, and M. V. Andres, Am. J. Phys. 61, 165 (1993). 